\title{
UNDERSTANDING BOX WING AIRCRAFT: ESSENTIAL TECHNOLOGY TO IMPROVE SUSTAINABILITY IN THE AVIATION INDUSTRY
}

\author{
Alexander SOMERVILLE, Matthew MARINO, Glenn BAXTER, Graham WILD \\ School of Aerospace Mechanical and Manufacturing Engineering, RMIT University, \\ Melbourne, Australia \\ E-mail: graham.wild@rmit.edu.au (corresponding author)
}

Received 31 October 2014; accepted 16 March 2016

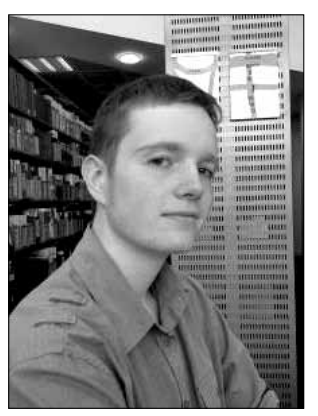

Alexander SOMERVILLE, Commercial Pilot

Education: 2010-2013 - Bachelor of Aviation, Edith Cowan University. 2012-2014-

Graduate Diploma in Flying, Edith Cowan University. 2012-2014 - Commercial Pilot Licence (Aeroplane), Advanced Cockpit Flight Training. 2014 - Flight Instructor Rating (Aeroplane), Advanced Cockpit Flight Training.

Research interest: advanced airframe concepts, sustainable aviation, aerospace vehicle efficiency, advanced aerospace vehicles.

Publications: author of 2 scientific article, 1 conference paper.

Present positions: 2015 - Sessional Academic, RMIT University, School of Engineering. 2016 Flight Instructor and Pilot, Avia Aviation.

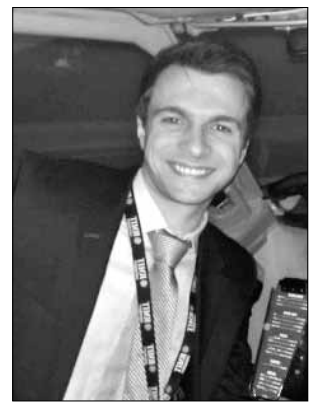

Matthew MARINO, PhD Aero Eng.

Education: 2003-2008 - Bachelor of Aerospace Engineering (Honours), RMIT University, 2009-2013 - PhD Aerospace Engineering, RMIT University.

Affiliations and functions: 2014-2015 - Post Doctorial Researcher, Sir Lawence Wackett Aerospace Research Center, RMIT University.

Research Interests: Sustainable aviation, aircraft systems, stabilization systems, aircraft performance, aviation technology, UAVs, UAV operations, drones, turbulence mitigation. Publications: author of 11 Articles, 3 book chapters, 16+ conference papers, 6 technical industry reports.

Present Position: 2016 - Lecturer in Aviation and Aerospace Engineering, RMIT University, School of Engineering.

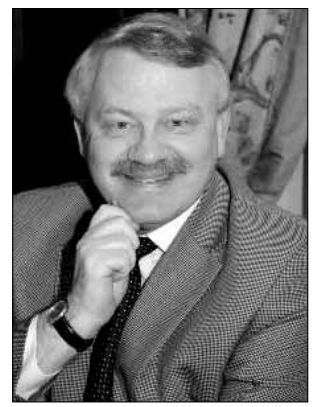

Glenn S. BAXTER, PhD (Aviation)

Education: Bachelor of Aviation Studies, University of Western Sydney, Australia, 2000. Master of Aviation Studies, University of Western Sydney, Australia, 2002. PhD, School of Aviation, Griffith University, Brisbane, Australia, 2010.

Affiliations and functions: Lecturer in Aviation Management and Deputy Program Manager Onshore Aviation Programs, RMIT University, School of Engineering.

Research interest: Air cargo handling and operations, airport operations, sustainable aviation, supply chain management.

Publications: Author of 15 scientific articles, 6 conference papers.

Present position: 2013 - Deputy Aviation Program Manager, RMIT University, School of Engineering. 


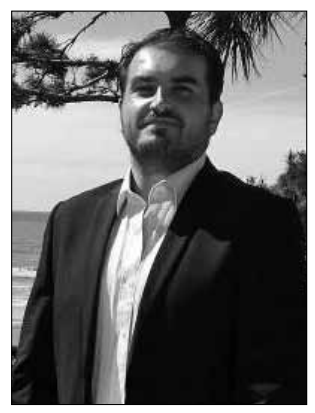

Graham WILD, PhD Eng.

Education: 2001-2004 - Bachelor of Science (Physics and Mathematics), Edith Cowan University. 2004-2005 - Bachelor of Science Honours (Physics), Edith Cowan University. 2008 - Graduate Certificate (Research Commercialisation), Queensland University of Technology. 2006-2008 - Master of Science and Technology (Photonics and Optoelectronics), the University of New South Wales. 2006-2010 - PhD (Engineering), Edith Cowan University. Affiliations and functions: 2010-2010 - Postdoctoral research associate, Photonics Research Laboratory, Edith Cowan University. 2011-2012 - Lecturer of Aviation Systems, Edith Cowan University. 2012 - Senior Lecturer in Aerospace and Aviation, RMIT University, School of Engineering. Research interest: Aircraft Systems, Aerospace Vehicle Health Monitoring, Optical Fibres, Sensing and Instrumentation, Sustainable Aviation, Aviation Technology. Publications: author of 22 articles, 4 book chapters, 60+ conference presentations. Present position: 2013 - Onshore Aviation Program Manager, RMIT University, School of Engineering.

\begin{abstract}
This paper presents an introduction to box wing aircraft technology, and an overview of current research efforts in this important area. Box wing technology offers a means of significantly reducing aircraft fuel consumption and hence improving economic sustainability. The result of this reduced fuel burn is a reduction in aircraft emissions, which will improve the environmental sustainability of the global aviation industry. This is important, because the industry has set an ambitious goal of reducing the current carbon emissions by 50\% before 2050, in the face of continued exponential growth in demand. The motivation of this work is to ensure that sufficient education is provided at all levels of the aviation industry, to keep people informed, and to help them make decisions. That is, there is confusion about the fundamental principles involved in the aerodynamic improvements associated with these innovative aircraft configurations. As such, this work presents box wing technology in the context of the fundamental operational aerodynamics associated with their implementation, giving an understanding of the performance benefits associated with them, in addition to the other practical benefits associated with box wing configurations.
\end{abstract}

Keywords: aircraft, box wing, Prandtl plane, joined wing, induced drag.

\section{Introduction}

To ensure the sustainability of the aviation industry, future aircraft will need to be more efficient, have lower direct operating costs, carry greater payloads, and continue to operate from current airports. These requirements will see the aviation industry be more sustainable economically and environmentally. To realise these requirements, the use of non-conventional and innovative aircraft technologies will be needed. Of significant interest is the concept of innovative aircraft configurations. The use of innovative aircraft configurations to improve aircraft performance characteristics is a concept that dates back to the Wright brothers, and is therefore a fundamental part of the aviation industry. Exploration of these non-conventional concepts has been pursued consistently since the earliest aircraft, and, as the conventional configuration reaches its technical limits, companies and individuals are continuing this research. Currently a number of innovative aircraft configurations are receiving attention. This includes the very high aspect ratio truss braced wing (Gur et al. 2011), or the flying wing style of the blended wing body aircraft (Liebeck 2004), and the box wing (Jemitola, Fielding 2012).

The box wing (also referred to as the Prandtl Plane, or joined wing), detailed by Ludwig Prandtl in his 1924 publication (Prandtl 1924), has the potential to decrease induced drag and increase lifting capabilities, without fundamentally increasing the aircraft's dimensions. We can see then that this meets all of the requirements stated above, in contrast to the other innovative aircraft configurations. In practice, this design could potentially have other advantages and disadvantages including altered pilot handling characteristics. In his work, Induced Drag of Multiplanes, Prandtl (1924) investigated lifting systems to find one with the minimum possible induced drag. At the time it was understood that as the number of vertically offset wing approached infinity, the induced drag of the lifting system tended towards zero. The solution to this problem was to join two vertically offset lifting surfaces at the wingtips, with horizontal aerodynamic structures. This solution results in substantially reduced induced drag, as compared with a monoplane configuration. Another advantage of this configuration is the distribution of the uplift requirement between two lifting surfaces, allowing for increased payloads within the constraints of current airports. Subsequent research has focused on the application of the box-wing concept to transport category aircraft, though there is some specific research into applications in the GA (general aviation) category.

In this paper, we present a complete and concise overview of box wing technology for the wider aviation community. This is especially important from an educational point-of-view, given that there is confusion about the fundamental principles involved. It is therefore intended that this work present box wing technology in the context of the fundamental aerodynamics associated with it. 


\section{Background}

In 2001 (Argüelles et al. 2001), the European Commission, in European Aeronautics: A Vision for 2020, set out the requirements for future civil aircraft. They concluded that aircraft in the coming decades would have to produce fewer noxious emissions, be quieter, and have reduced direct operating costs. In addition, aircraft of the future should be capable of operating from current airports as well as meeting other safety and security preferences (Argüelles et al. 2001). The environmental improvements set forth by the European Commission are in accordance with the reductions recommended by the Intergovernmental Panel on Climate Change (Penner et al. 1999), and the hopes of the International Civil Aviation Organisation. The box wing concept has the potentialto achieve these outcomes, and research has progressed to considering its viability as compared to a conventional cantilever configuration for a wing.

Since the 1960's, increased aircraft efficiency has come from a combination of improved engine design, enhanced structural aerodynamics, and new materials. Modern aircraft, for example, are $70 \%$ more efficient than aircraft in the 1960's (Penner et al. 1999). Industry led efficiency improvements are likely to continue into the future; however, the conventional airframe configuration appears to be reaching an efficiency plateau. Therefore, as the required emission reductions cannot come completely from engine and aircraft material improvements, the box-wing may offer a potential solution.

\section{Aerodynamic considerations}

\subsection{Span wise flow}

First we must think about how a wing develops lift (Hurt 2012). As with all modern literature, we must dismiss the need to invoke the "equal transit time theory" (air travelling over the top surface must speed up to reach the trailing edge at the same time as flow travelling under the bottom surface). Instead, we simply note that the static air pressure over the top surface will be less than the static air pressure under the bottom surface (both will typically be less than atmospheric pressure, depending on the aerofoil shape). This pressure difference results in a net force lifting the aircraft upwards.

If we have a net lift, we know we must have a lower pressure on the upper surface relative to the bottom surface. As a result, when we have wing tips, the pressure difference will result in flow from the lower surface to the upper surface around the wing tips. That is, typically we think of flow from the leading edge to the trailing edge, but flow can easily move around the wing tip as well. This concept is shown in Figure 1. The result of this is that the flow along the upper and lower surfaces will not be linear from the LE to the TE, but rather there will also be a span wise component of the flow. That is, on the bottom surface of the wing, air will tend to flow from root to tip, while on the top surface of the wing, air will tend to flow from tip to root (Anderson 2011a).

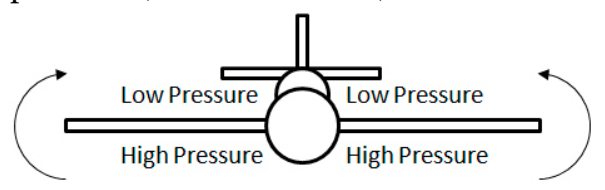

Fig. 1. Tip flow for an aircraft wing when producing lift

Figure 1 also highlights that the flow about one wing tip is the opposite relative to the other wing tip. To facilitate this condition, the flow in the middle of the wing must be neither flowing port nor starboard relative to the aircraft. That is, the span wise flow, or flow along the wing span, varies from wing tip to wing tip. Figure 2 shows that the opposing span wise flow will result in a span wise component that is zero in the middle of the wing, and maximum at the tips.

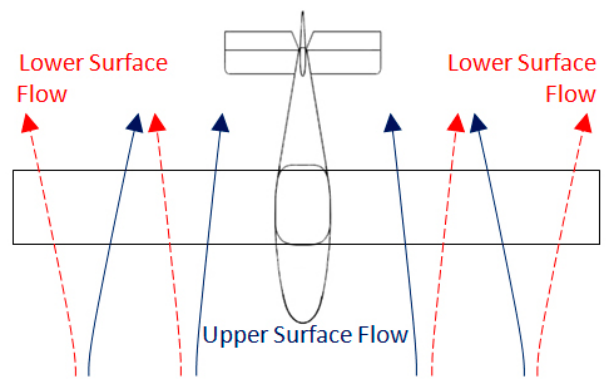

Fig. 2. Tip flow for an aircraft wing when producing lift

\subsection{Wing tip vortices}

The rotating flow around the wing tips, coupled with the free-stream flow, results in a vortex. This flow is called a wing tip vortex.

The span wise flow will also be a function of the lift generated. More lift means a greater pressure differential. This results in a more span wise flow. The greater the span wise flow the larger the vortices will be (Kermode et al. 2012). Figure 3 shows an aircraft with visible condensation in the core of the wing tip vortices, giving a direct visual indication of their presence.

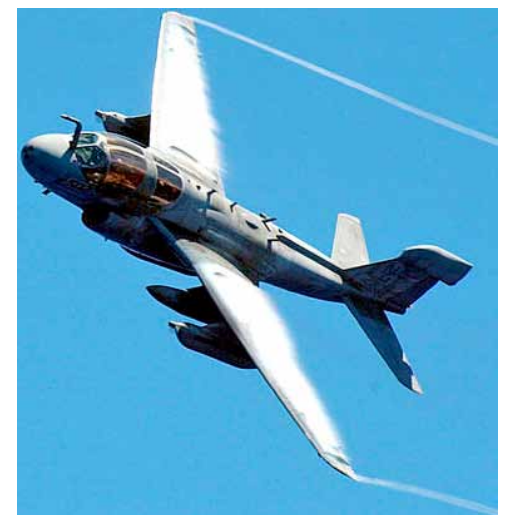

Fig. 3. Condensation in the core of the wing tip vortices of an aircraft (source: United States Navy 2003) 


\subsection{Induced flow}

A result of the vortices from the TE and wingtips is the induced circulation of the flow along the chord giving a vertical component to the flow. This is upwash ahead of the wing, and downwash aft of the wing. The downwash behind the wing is such that a fixed velocity is imparted to the flow by a wing producing lift.

The result of this induced flow is that the wing is actually in a relative flow that is inclined downward. So the angle of attack will not simply be the angle between the free-stream flow (far away from the wing) and the chord line of the wing. We now must consider the downwash effect, such that the angle of attack, as traditionally defined, is greater than the effective relative angle of attack (between the chord and local flow). As such a larger angle of attack is needed to provide the additional lift to make up for the downwash.

\subsection{Induced drag}

The result of this induced flow is that the lift vector is now inclined at an angle, and only a vertical component is effective in the production of lift. So, the horizontal component of the lift is pointing back, opposite to the thrust vector, hence this must be a drag component. This horizontal lift component is a drag component, and it is called induced drag $\left(D_{\mathfrak{i}}\right)$. It is the drag induced by the generation of lift from a finite wing (Anderson 2011b). This is shown in Figure 4.

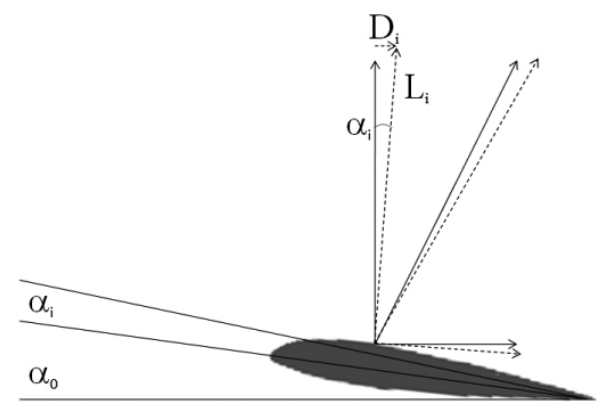

Fig. 4. Induced flow at the total angle of attack requires the aerodynamic reaction force to be longer, increasing the length of the relative vertical component

Since induce drag is directly related to lift, then the coefficient of induced drag is related to the coefficient of lift. They are related by the induced angle of attack $\left(\alpha_{\mathrm{i}}\right)$. We can use the standard equation for an aerodynamic force, relating the wing surface area $(S)$, dynamic pressure (q), and coefficient to the force. For induced drag this will be:

$$
D_{i}=C_{D} q S \text {. }
$$

Assuming we have a small induced angle of attack $\left(\boldsymbol{\alpha}_{\mathrm{i}}\right)$, less than 10 degrees, we can use the small angle approximation. That is, in a triangle with a small angle, the longest side is approximately equal to the hypotenuse. From Figure 4, the triangle shown for the lift components relates the Lift force $\left(L_{i}\right)$ to the induced drag for $\left(D_{i}\right)$.
Incorporating (1) we can use the corresponding coefficients in our trigonometric relationship, which gives:

$$
C_{D i}=C_{L} \sin \alpha_{i} \text {. }
$$

For an elliptical wing, which will result in an elliptical pressure distribution, this becomes:

$$
C_{D i}=\frac{C_{L}^{2}}{\pi A R} .
$$

Recall that the aspect ratio $(A R)$ is the ratio of the wing span to the chord length $(\mathrm{AR}=\mathrm{b} / \mathrm{c})$. Combining the last two equations, (2) and (3), gives us:

$$
C_{L} \sin \alpha_{i}=\frac{C_{L}^{2}}{\pi A R} .
$$

Using radians for the angle, the small angle approximation gives:

$$
\alpha_{i}=\frac{C_{L}}{\pi A R} .
$$

This is an important equation as it shows that the induced angle of attack is directly proportional to the coefficient of lift (and hence angle of attack), and inversely proportional to the aspect ratio. That is, flight in high lift conditions with low speed will give a high induced angle of attack, and hence high induced drag, while high speeds will result in low induced angles of attack. Logically, this makes sense, as the high lift conditions should result in the greatest amount of downwash.

Note that if we have an infinite wing, the AR will be infinite, so the induced angle of attack is zero for an aerofoil. Conversely, a very small wing span will have a small aspect ratio giving a large induced angle of attack.

\section{Induced drag factor}

The above description assumes a wing has an elliptical wing planform with a corresponding elliptical lift distribution, as shown in Figure 5. In general, if we have a wing that is not elliptical, then the coefficient of induced drag becomes (Anderson 2011b):

$$
C_{D i}=\frac{C_{L}^{2}}{\pi e A R} .
$$

The new term in the denominator is referred to as the Oswald Efficiency Factor (e). For most conventional wing geometries this will be less than or equal to $1(\mathrm{e} \leq 1)$. For an elliptical wing it is equal to 1 . As a result, an elliptical wing for a given aspect ratio will have the lowest induced drag, assuming we have a conventional monoplane.
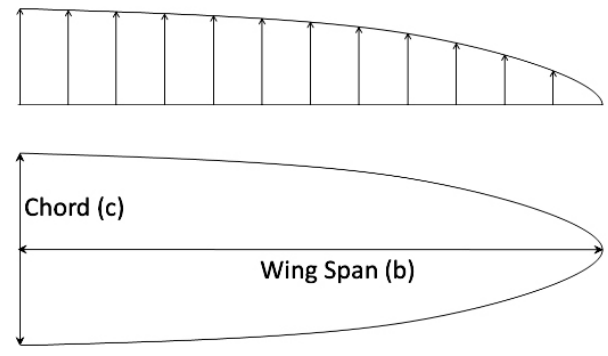

Fig. 5. Elliptical wing semispan with a corresponding elliptical lift distribution 
As we are working with induced drag, which we are trying to minimise, the Oswald Efficiency Factor can be slightly confusing. That is, a larger Oswald Efficiency Factor results in less drag, since $e$ is in the denominator. As such, it is more convenient to work with the induced drag factor $(\delta)$. When using this, the induced drag coefficient becomes (Anderson 2011b):

$$
C_{D i}=\frac{C_{L}^{2}}{\pi A R}(1+\delta) \text {. }
$$

From (6) and (7) we can see that the relationship between the Oswald Efficiency Factor and the induced drag factor is:

$$
\frac{1}{e}=(1+\delta) \text {. }
$$

Typically, the drag factor will be greater than or equal to $0(\delta \geq 0)$. For an elliptical wing the drag factor is 0 .

\subsection{Tapered wings}

The discussion above referred to the low induced drag of the elliptical wing planform, due to the induced drag factor of 0 . For tapered wings, the induced drag factor varies as a function of the aspect ratio and the taper ratio (the ratio of the chord at the wing tip relative to the chord at the wing root). Figure 6 shows how the induced drag factor varies as a function of taper ratio and aspect ratio.

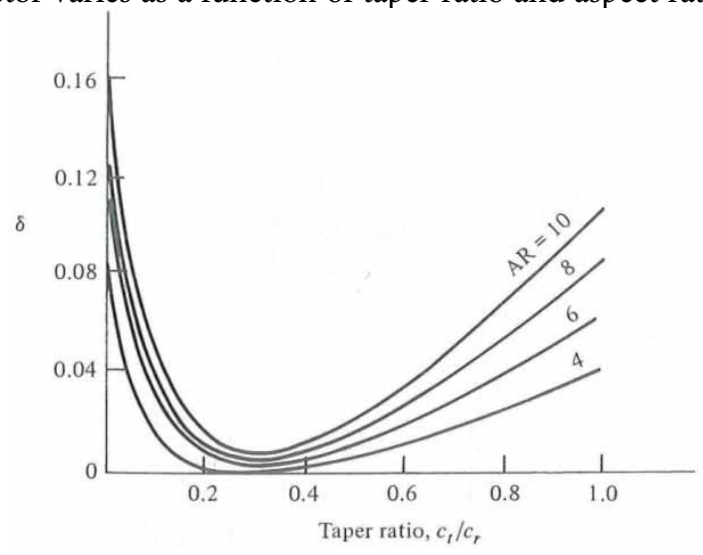

Fig. 6. Induced flow at the total angle of attack requires the aerodynamic reaction force to be longer, increasing the length of the relative vertical component

From Figure 6 we see that it is possible to have a quadrilateral semispan with a drag factor of 0 ; this occurs with an aspect ratio of 4 and a taper ratio of approximately 0.3.

\subsection{Non-planar wings}

Now we can consider non-planar wing geometries. These configurations are shown in Figure 7. First we see that even a small change of the geometry in the vertical direction has an effect. That is, the upper left figure shows a wing with dihedral, with an induced drag factor of -0.029 . This can be improved substantially if we go to wing tip modifications, as seen in the third left figure and the second, third, and forth right figures of Figure 7.
We can also see the benefit of a biplane structure, and hence the inspiration for Prandtl's original investigation. This is then minimised with the box wing structure, illustrated in the bottom right figure of Figure 7.

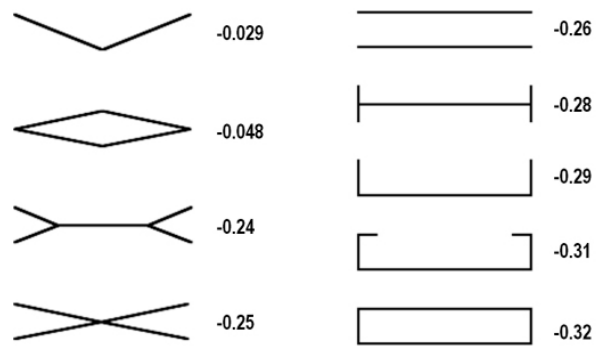

Fig. 7. Induced flow at the total angle of attack requires the aerodynamic reaction force to be longer, increasing the length of the relative vertical component

From Figure 7 we can see that the box wing has an induced drag factor of -0.32 ; that is, a $32 \%$ reduction in the induced drag. There will be a corresponding reduction in total drag, and therefore an improvement to lift-to-drag ratio. The overall consequence of implementing a box wing will be improvements in range and endurance, or reductions in fuel consumption. There is also a greater wing volume giving greater fuel storage, again giving further improvements to range and endurance. Another advantage of the box wing aircraft is that it has a conventional fuselage (eliminating structural issues associated with blended wing body aircraft), and this also means that conventional airport gates can be used without modification.

\section{State of the art}

The application of the box-wing to large and medium sized aircraft was shown to have many of the required characteristics (Frediani 2005). For example, the positive effects of the reduction of induced drag (reduced noxious emissions). Box-wing aircraft of this size and configuration have numerous aerodynamic, comfort and ground-operation advantages, compared to a conventional configuration. It was also concluded that there are safety benefits, including better pitch control and reduced vortex formation. In addition, the aircraft would be capable of carrying more cargo, or be configured to carry up to 600 passengers. As a result of these capabilities, there are numerous economic benefits beyond fuel savings.

The application of the box-wing concept to small aircraft was also examined, with positive conclusions. A small box-wing aircraft would likely be safer, due to the position of occupants within the structure and the improved structure itself. It was also suggested that such an aircraft would be highly stable, and be extremely aerodynamically efficient. Though positive, Frediani (2005) concluded that more research is required for both small and large aircraft. 
Voskuijl et al. (2012) investigated the feasibility of a 300 passenger box-wing aircraft. This investigation focused on the propulsion system and control systems of such an aircraft. An initial design for the aircraft was then created for use in the preliminary design of these systems.

The result of this investigation was a box-wing aircraft with two conventional turbofan engines, mounted at the rear tail of the aircraft. In this investigation, like in most box-wing research, control surfaces are distributed across both wings. This configuration gives two advantages, as compared to a conventional aircraft. If the control surfaces are differentially deflected, "a pure moment can be created" (Voskuijl et al. 2012). Alternatively, a combined deflection of both control surfaces "allows the use of direct lift control". The investigation also found that the box-wing aircraft exhibited good longitudinal control characteristics, though was slightly unstable under certain circumstances.

Any commercially viable, and operator accepted aircraft, would have to utilise some form of high lift system. Iezzi (2006) analysed the low-speed aerodynamics of a given box-wing; in relation to high lift systems. Firstly, a study of the current design was conducted to identify trends and guidelines in order to determine what was achievable. The "PP250" concept, developed by Bottoni and Scanu (2004), was a primary consideration of this paper. The requirements of the European Commission's future aircraft, as set out in European Aeronautics: a vision for 2020 (Argüelles et al. 2001), were also considered. A new set of tools and techniques for the prediction of performance and analysis of design modifications were consequently developed.

The conclusions of this research are positive, though it is clear that more re-search will be required. New methods for the analysis of box-wing aircraft were developed and validated. Analysis of the PP250 was undertaken, in order to improve stalling characteristics, with changes in wing washout being applied. The research also analysed the effectiveness of flap configurations, finding that the best configuration would be a single-slotted Fowler flap on the forward wing, and a double-slotted Fowler flap on the rear wing. Though this conclusion was found when analysing a large aircraft, it is possible that a similar result would be found for a smaller aircraft.

The paper made a number of recommendations for future research, in relation to high lift systems, flaps and box-wings. One suggestion is further wind tunnel testing, to gather experimental data about box-wings with flaps. Another suggestion is the investigation of boxwings at takeoff, due to potential reductions in drag. A further suggestion is that a box-wing, and conventional, aircraft be concurrently designed for the same mission, using the same tools. This would potentially show a more accurate comparison, and highlight any advantages of the box-wing concept.
Van Ginneken et al. (2010) presented a methodology for the design of the primary flight control surfaces for a fixed wing aircraft. The specific example explored was a box-wing aircraft, with a passenger capacity of 300. The results show good handling characteristics, and, much like Voskuijl et al. (2012), suggest the possibility of pure pitching and direct lift control.

Research into the application of the box-wing concept to small aircraft is also being undertaken. Synergy Aircraft are currently designing a new aircraft, based on the work of Prandtl (1924), which they called the "double boxtail configuration" (Synergy Aircraft 2014). The aircraft is one of a few box-wing concepts being developed for the General Aviation (GA) category, a market, which is primarily controlled by the Cessna C172 "Skyhawk". The new aircraft is claimed to have aerodynamic drag reduced to the Gabriell-von Karman limit, under certain circumstances. The configuration visual appears closer to a c-wing, and has some design similarities to the joined-wing concept. The aircraft is currently undergoing scaled-model testing, and could potentially be powered by a turbo-diesel engine. However, the US patent for the concept, which had been publicly available, is no longer online. The reason for this is not clear; however, it is possible that it infringes upon other boxwing or joined-wing patents.

Future aircraft will be required to be more efficient, safer, have lower direct operator costs, carry greater payloads and be capable of operating in current airports. The European Commission, Intergovernmental Panel on Climate Change (IPCC) and the International Civil Aviation Organization (ICAO) have all accepted that there must be emission reduction, and each has set out their requirements for future civil aircraft. Ludwig Prantdl's box-wing configuration offers a potential solution, and research is currently being undertaken to evaluate the viability. Individuals, academia and industry are undertaking this research around the world.

The research presented in this paper represents only a portion of the current, and past, research into boxwing aircraft. However, the research indicates that the opportunity exists for further research into this potentially innovative aviation design concept. Box-wing aircraft design has the potential to reduce the induced drag, and therefore emissions bringing both environmental and economic benefits to an increasing diverse global aviation industry.

\section{Analytical simulation}

The benefits of the box wing design can be evaluated by comparing the thrust and power requirements between a conventional and box wing configuration on an arbitrary aircraft. In this instance, we take the Cessna 172 classic aircraft as the subject. Data relating to the study are featured in Table 1. 
In this study we assume a constant weight and altitude at $5000 \mathrm{ft}$ above sea level to simulate a common cruise flight profile. The velocity variation is in accordance with the aircraft's performance capabilities to give an indication on the changes in power and thrust throughout its entire operational flight envelope. The thrust and power required are calculated in the normal manner using (8) and (9), which assumes that thrust equals drag for a level and unaccelerated flight profile. The drag factors in this analysis are $\delta=-0.029$ for the conventional configuration, and $\delta=-0.32$ for the box wing configuration (refer to Fig. 7).

Table 1. Relevant specifications of the Cessna 172 classic

\begin{tabular}{|l|l|}
\hline Aircraft & Cessna 172 classic \\
\hline Weight (Max GTOW) & $1,111 \mathrm{~kg}$ \\
\hline Wing Area & $17.2 \mathrm{~m}^{2}$ \\
\hline Density of air @ 5000ft & $0.996 \mathrm{~kg} / \mathrm{m}^{3}$ \\
\hline Velocity Range & $25-100 \mathrm{~ms}^{-1}$ \\
\hline Aspect Ratio & 7.32 \\
\hline Parasite drag Coefficient & 0.02 \\
\hline
\end{tabular}

$$
\begin{aligned}
T_{R}=D & =q_{\infty} S\left[C_{D_{o}}+\frac{C_{L}^{2}}{\pi A R}(1+\delta)\right], \\
P_{R} & =T_{R} V_{T A S} .
\end{aligned}
$$

The box-wing configuration displays a significant reduction in the amount of thrust required for steady level flight. The reduction in induced drag is a direct consequence of the reduced drag factor. It is logical to assume that significant differences between the conventional and box wing configurations are found at velocities where induced drag significantly contributes the overall aircraft drag. This is evident in Figure 8, where the majority of the thrust savings between 7 and $28 \%$ are found in the lower flight velocities, between 25 and 60 $\mathrm{ms}^{-1}$. Velocities greater than $60 \mathrm{~ms}^{-1}$ display less thrust reduction as the drag factor and induced drag become less influential and parasite drag begins to dominate.

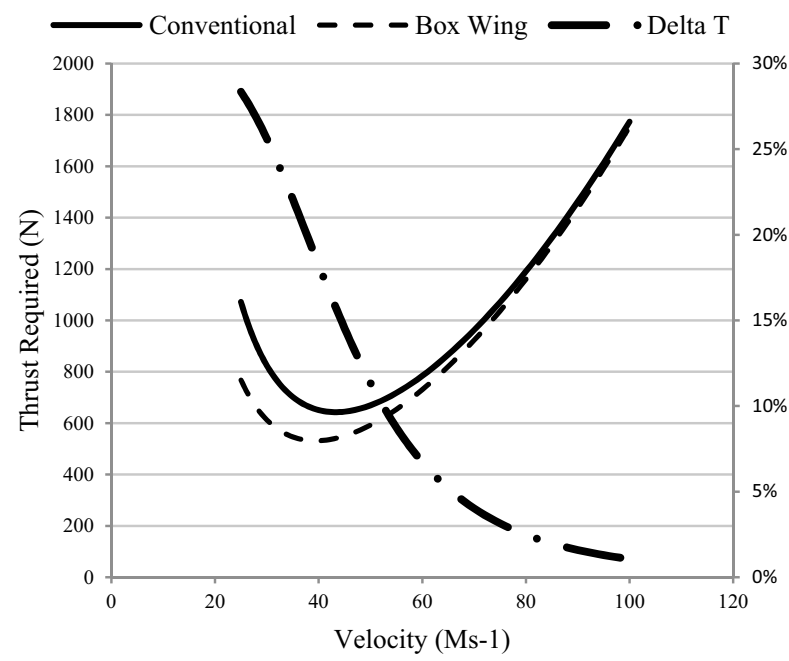

Fig 8. Thrust required versus aircraft TAS for a Cessna 172

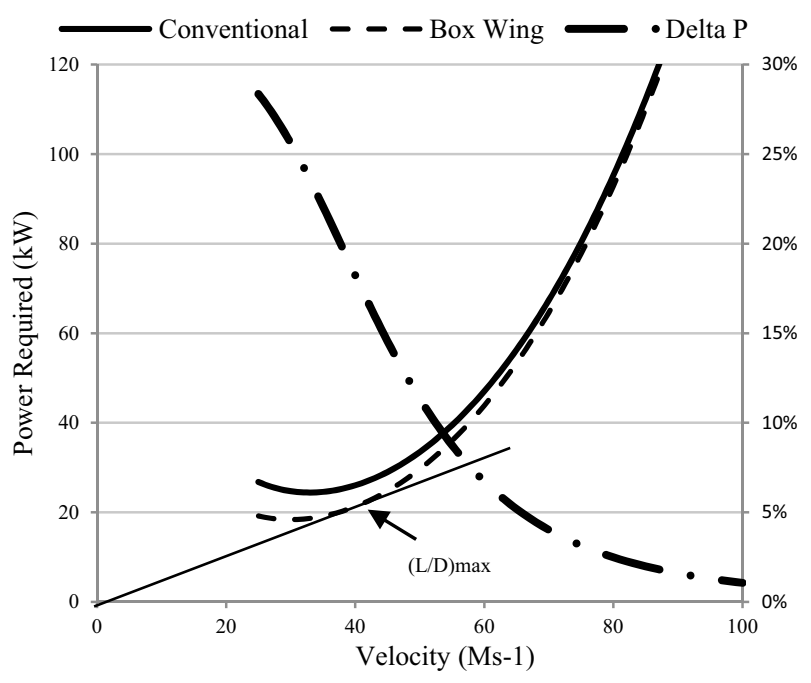

Fig. 9. Power required versus aircraft TAS for a Cessna 172

The power required follows similar trends to those found previously for the Thrust required. Significant reductions in the power required are found at the lower flight velocities. It must also be noted that the box-wing configuration requires less thrust and power across the velocity range thus improving the aircraft's efficiency and flight performance.

The box-wing configuration enhances the aircraft's range by reducing the minimum thrust required for level unaccelerated flight by approximately $18 \%$. This is where the maximum lift/drag ratio $\left((\mathrm{L} / \mathrm{D})_{\max }\right)$ exists. The tangent to the power required curve locates the point of the minimum thrust required (refer to Fig. 9). Furthermore, an aircraft'ss endurance is also expanded as a result of reducing minimum power by approximately $24 \%$. This translates to significant fuel savings as thrust, power and fuel consumption are linearly correlated.

\section{Conclusions}

In conclusion, we have presented box-wing technology. An attempt has been made to work from fundamental principles, to ensure that a wider audience in the aviation industry clearly understands the technology. We have also presented a short overview of research efforts into box-wing aircraft available in the literature.

We have presented the numerous advantages of box-wing technology; specifically, the fact that its use will offer significant reductions in aircraft fuel consumption,. The by-product of the reduction in fuel consumption is a reduction in aircraft emissions; this will therefore improve the environmental sustainability in the aviation industry.

\section{References}

Argüelles, P.; Bischoff, M.; Busquin, P., et al. 2001. European aeronautics: a vision for 2020. European Commission [online], [cited 19 December 2014]. Available from the Inter- 
net: http://ec.europa.eu/research/growth/aeronautics2020/ pdf/aeronautics2020_en.pdf

Anderson, J. D. 2011a. Introduction to flight. New York: McGraw-Hill.

Anderson, J. D. 2011b. Fundamentals of aerodynamics. New York: McGraw-Hill.

Bottoni, C.; Scanu, J. 2004. Preliminary design of a 250 passenger PrandtlPlane aircraft: Unpublished Graduating thesis in Aerospace Engineering. University of Pisa.

Frediani, A. 2005. The Prandtl Wing, in V K I, Lecture series: "Innovative configurations and advanced concepts for futurecCivil transport aircraft", 06-10 June 2005 [online], [cited 19 December 2014]. Available from the Internet: http://www.engbrasil.eng.br/index_arquivos/art95.pdf

Gur, O.; Schetz, J. A.; Mason, W. H. 2011. Aerodynamic considerations in the design of truss-braced-wing aircraft, Journal of Aircraft 48(3): 919-939. http://dx.doi.org/10.2514/1.C031171

Hurt, H. H. 2012. Aerodynamics for naval aviators. New York: Skyhorse Publishing.

Iezzi, G. 2006. PrandtlPlane high lift system preliminary aerodynamic design: Unpublished thesis. Università Di Pisa, Italy [online], [cited 19 December 2014]. Available from the Internet: https://etd.adm.unipi.it/theses/available/etd09222006-113301/unrestricted/tesi_iezzi.pdf

Jemitola, P. O.; Fielding, J. P. 2012. Box-wing aircraft conceptual design, in the 28th International Congress of the Aeronautical Sciences, ICAS, 2012 [online], [cited 19 December 2014]. Available from the Internet: http://www.icas.org/ ICAS_ARCHIVE/ICAS2012/PAPERS/213.PDF

Kermode, A. C.; Barnard, R. H.; Philpott, D. R. 2012. Mechanics of flight. New Jersey: Pearson Education.
Kroo, I. 2001. Drag due to lift: concepts for prediction and reduction, Annual Review of Fluid Mechanics 33(1): 587-617. http://dx.doi.org/10.1146/annurev.fluid.33.1.587

Liebeck, R. H. 2004. Design of the blended wing body subsonic transport, Journal of Aircraft 41(1):10-25. http://dx.doi.org/10.2514/1.9084

Penner, J. E.; Lister, D. H.; Griggs, D. J., et al. 1999. Aviation and the global atmosphere. Cambridge, UK: Cambridge University Press.

Prandtl, L. 1924. Induced drag of multiplanes. Washington, DC: National Advisory Committee for Aeronautics.

Synergy Aircraft. 2014. Quiet, roomy, and fuel efficient: synergy is a new class of aeroplane [online], [cited 19 December 2014]. Available from Internet: http://synergyaircraft.com/

United States Navy. 2003. EA-6B Prowler from VAQ-138.jpg [online], [cited 19 December 2014]. Available from Internet: http://commons.wikimedia.org/wiki/File:EA-6B_ Prowler_from_VAQ-138.jpg

van Ginneken, D. A. J.; Voskuijl, M.; van Tooren, M. J. L., et al. 2010. Automated control surface design and sizing for the Prandtl Plane, in the 51st AIAA/ASME/ASCE/AHS/ASC Structures, Structural Dynamics, and Materials Conference 18th, 12-15 April 2010, Orlando, Florida [online], [cited 19 December 2014]. Available from the Internet: http://enu. kz/repository/2010/AIAA-2010-3060.pdf

Voskuijl, M.; De Klerk, J.; Van Ginneken, D. 2012. Flight mechanics modeling of the PrandtlPlane for conceptual and preliminary design, in G. Buttazzo, A. Frediani (Eds.). Variational analysis and aerospace engineering: mathematical challenges for aerospace design. New York: Springer Science+Business Media, LLC, 435-462. 